\title{
DALY Estimation Approaches: Understanding and Using the Incidence-based Approach and the Prevalence-based Approach
}

\author{
Young-Eun Kim ${ }^{1}$, Yoon-Sun Jung ${ }^{2}$, Minsu Ock ${ }^{3,4}$, Seok-Jun Yoon ${ }^{5}$ \\ ${ }^{1}$ Department of Big Data Strategy, National Health Insurance Service, Wonju, Korea; ${ }^{2}$ Institute for Future Public Health, Graduate School of Public \\ Health, Korea University, Seoul, Korea; ${ }^{3}$ Department of Preventive Medicine, University of Ulsan College of Medicine, Seoul, Korea; ${ }^{4}$ Department of \\ Preventive Medicine, Ulsan University Hospital, University of Ulsan College of Medicine, Ulsan, Korea; ${ }^{5}$ Department of Preventive Medicine, Korea \\ University College of Medicine, Seoul, Korea
}

Disability-adjusted life-year (DALY) estimates may vary according to factors such as the standard life expectancy, age weighting, time preference and discount rate, calculation of disability weights, and selection of the estimation method. DALY estimation methods are divided into the following 3 approaches: the incidence-based approach, the pure prevalence-based approach, and the hybrid approach. These 3 DALY estimation approaches each reflect different perspectives on the burden of disease using unique characteristics, based on which the selection of a suitable approach may vary by the purpose of the study. The Global Burden of Disease studies, which previously estimated DALYs using the incidence-based approach, switched to using the hybrid approach in 2010, while the National Burden of Disease studies in Korea still mainly apply the incidence-based approach. In order to increase comparability with other international burden of disease studies, more DALY studies using the prevalence-based approach need to be conducted in Korea. However, with the limitations of the hybrid approach in mind, it is necessary to conduct more research using a disease classification system suitable for Korea. Furthermore, more detailed and valid data sources should be established before conducting studies using a broader variety of DALY estimation approaches. This review study will help researchers on burden of disease use an appropriate DALY estimation approach and will contribute to enhancing researchers' ability to critically interpret burden of disease studies.

Key words: Disability-adjusted life-year, Prevalence, Incidence, Disability weight

\section{INTRODUCTION}

Among indicators of the burden of disease, disability-adjusted life-years (DALYs) are calculated as the sum of years of life

Received: November 10, 2021 Accepted: January 12, 2022

Corresponding author: Minsu Ock

Department of Preventive Medicine, Ulsan University Hospital,

University of Ulsan College of Medicine, 877 Bangeojinsunhwando-ro,

Dong-gu, Ulsan 44033, Korea

E-mail: ohohoms@naver.com

This is an Open Access article distributed under the terms of the Creative Commons Attribution Non-Commercial License (https://creativecommons.org/licenses/by$\mathrm{nc} / 4.0 /$ ) which permits unrestricted non-commercial use, distribution, and reproduction in any medium, provided the original work is properly cited. lost (YLL) and years lost due to disability (YLD) [1]. DALYs were first used in a global burden of disease (GBD) study in 1992 [2]. In that study, the GBD was analyzed based on DALYs by age, sex, and region, where diseases and injuries were divided into 21 groups and 107 diseases to measure DALYs for each disease and analyze the influence of 19 risk factors [3,4]. The study also provided predicted values up to 2020 based on DALY measurements in 1990 [5].

The DALY-based estimation of the burden of disease is important because it simultaneously considers the reduced health state due to disability before death and the decline in life expectancy due to death. It thus moves away from conventional measurements of the burden of disease that use readily available data 
on mortality, prevalence, and incidence. Indicators that simultaneously measure mortality and a decrease in the health state due to the prevalence or incidence of disease are known as summary measures of population health, and since the 1990 GBD study, DALYs have been used as a representative indicator of the burden of disease [6]. GBD studies were published in 1999, 2000, 2001, 2002, and 2004 [7]. DALYs have also been applied in national burden of disease (NBD) studies in 37 countries.

A number of factors should be considered to estimate DALYs. DALY estimates may vary according to factors such as the standard life expectancy, age weighting, time preference and discount rate, calculation of disability weights (DWs), and selection of the estimation method [8,9]. A significant breakthrough came in GBD studies with the selection of the estimation approach [10]. In contrast to the GBD studies in the 1990s and early 2000s, in which the incidence-based approach was applied, a 2010 GBD study introduced the concept of sequelae to measure the burden of disease of 1160 after-effects related to 291 diseases and injuries across 187 countries, as well as evaluating the effects of 67 risk factors [11,12].

In this study, the characteristics of and differences in the incidence-based and prevalence-based approaches to DALY es- timation are examined, and the use of each approach is assessed by comparing their advantages and disadvantages. This review of DALY estimation approaches contributes to the literature by facilitating more accurate measurements and thus enhancing the understanding and policy-making utility of DALYs.

\section{UNDERSTANDING DISABILITY-ADJUSTED LIFE-YEAR ESTIMATION APPROACHES}

To refine the aforementioned DALY estimation approaches, the prevalence-based approach is sub-categorized into the pure prevalence-based approach and the hybrid approach [13]. As each approach measures different phenomena, one is likely to be more useful than the other in specific circumstances. To understand each approach, a hypothetical case may be conjectured, as shown in Figure 1, which represents the life of an individual who contracts disease 1 at age 10, causing a DW of 0.25 , followed by disease 2 at age 50 , causing an additional DW of 0.4. The individual dies at 60 years, 20 years earlier than the life expectancy of 80 years. Using this hypothetical case, the DALY estimation using each approach is reviewed.

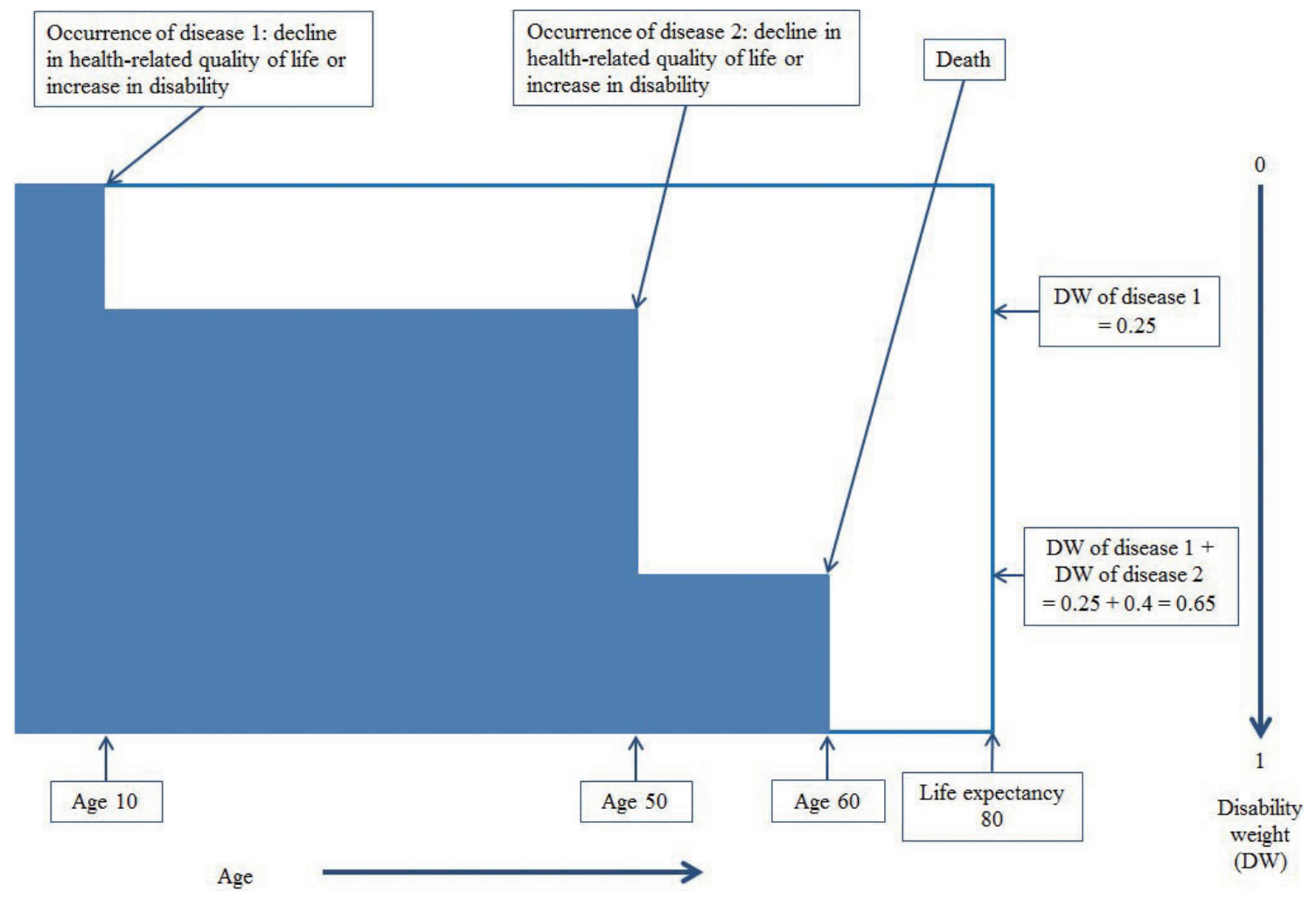

Figure 1. A hypothetical case to explain the disability-adjusted life-year estimation approaches. 


\section{Incidence-based Approach}

Figure 2 shows the DALY estimation using the incidence-based approach. The total YLD is the sum of YLD 1, corresponding to the product of the DW of disease $1(0.25)$ and the time between onset (10 years) and survival (60 years), and YLD 2, which is the product of the DW of disease $2(0.4)$ and the time between onset (50 years) and survival (60 years). YLL is obtained as the product of the DW of death (1.0) and the difference between the time of death (60 years) and life expectancy (80 years). DALY is the sum of YLD and YLL. As previously mentioned, factors such as the standard life expectancy, age weighting, time preference and discount rate, and DW should be considered $[8,9]$.

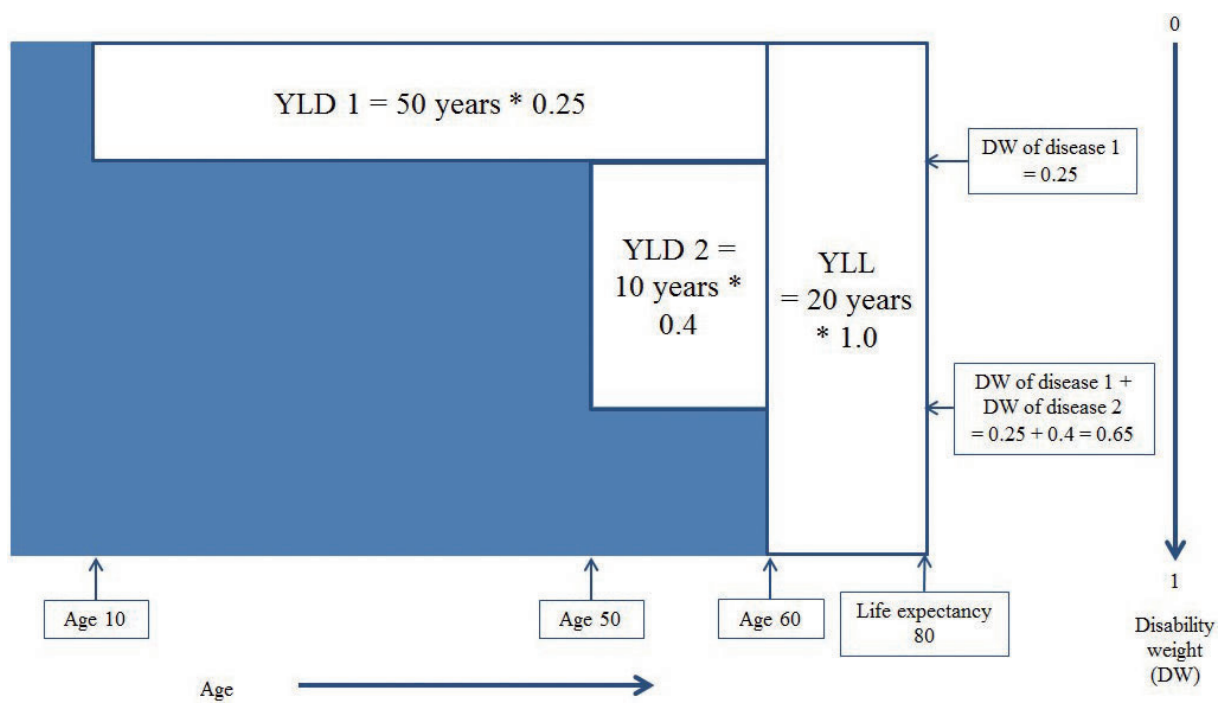

Figure 2. An example of disability-adjusted life-year estimation using the incidence-based approach. YLD, years lost due to disability; YLL, years of life lost.

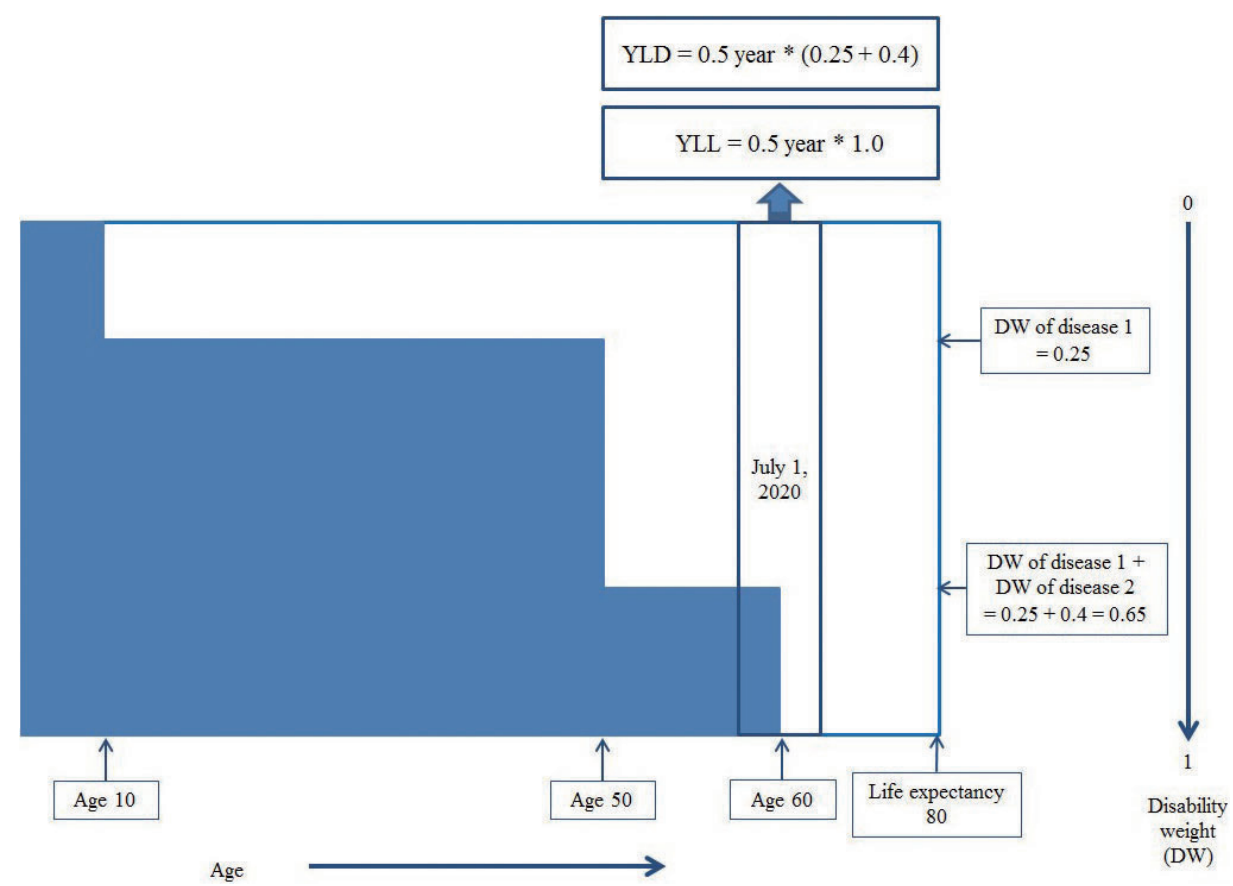

Figure 3. An example of disability-adjusted life-year estimation using the pure prevalence-based approach. YLD, years lost due to disability; YLL, years of life lost. 


\section{Pure Prevalence-based Approach}

Figure 3 shows the DALY estimation using the pure prevalence-based approach. In this approach, as the DALYs are estimated within a year, additional assumptions should be made to describe the overall estimation process. If an individual dies on July 1,2010, YLD is the product of half a year ( 0.5 years) and the sum of the DWs of disease 1 and disease $2(0.65)$, whereas YLL is the product of half a year ( 0.5 years) and the DW of death (1.0). The pure prevalence-based approach is characterized by measuring the burden of disease in the year of incidence instead of across the entire lifetime of an individual.

\section{Hybrid Approach}

The DALY estimation using the hybrid approach applies the prevalence-based approach to estimate YLD and the incidencebased approach to estimate YLL (Figure 4). YLD in this approach is the sum of the DWs caused by diseases 1 and $2(0.65)$ in 2020. YLL, as in the incidence-based approach, is the product of the DW of death (1.0) and difference between the time of death (60 years) and life expectancy (80 years). In accordance with the use of the accurate time of death of the individual, the YLL and YLD measurements may overlap, yielding an overestimation of DALYs; however, this is unlikely to be significant for DALY estimates for the total population.

\section{THE APPLICATION AND ADVANTAGES/ DISADVANTAGES OF DISABILITY-ADJUSTED LIFE-YEAR ESTIMATION APPROACHES}

These 3 DALY estimation approaches each reflect different perspectives on the burden of disease using unique characteristics. There is no absolute criterion for selecting an approach; instead, the selection of a suitable approach may vary according to the purpose of the study.

The pure prevalence-based approach may be more suitable for studies that focus on the burden of disease in a specific year. In addition, it can be used to roughly estimate the burden of non-fatal diseases such as allergic rhinitis. However, the burden of disease is typically estimated to determine the priority of certain policies and interventions or to evaluate their effects, and the pure prevalence-based approach is infrequently applied to estimate the burden of disease. The incidence-based approach, rather than the pure prevalence approach, is more helpful in determining whether measures should be taken to prevent the occurrence of a particular disease from a long-term perspective. For example, whether to introduce a human papillomavirus (HPV) vaccination program to reduce the burden of HPV-related cervical cancer may be more effectively determined using DALY estimates with the incidence-based approach.

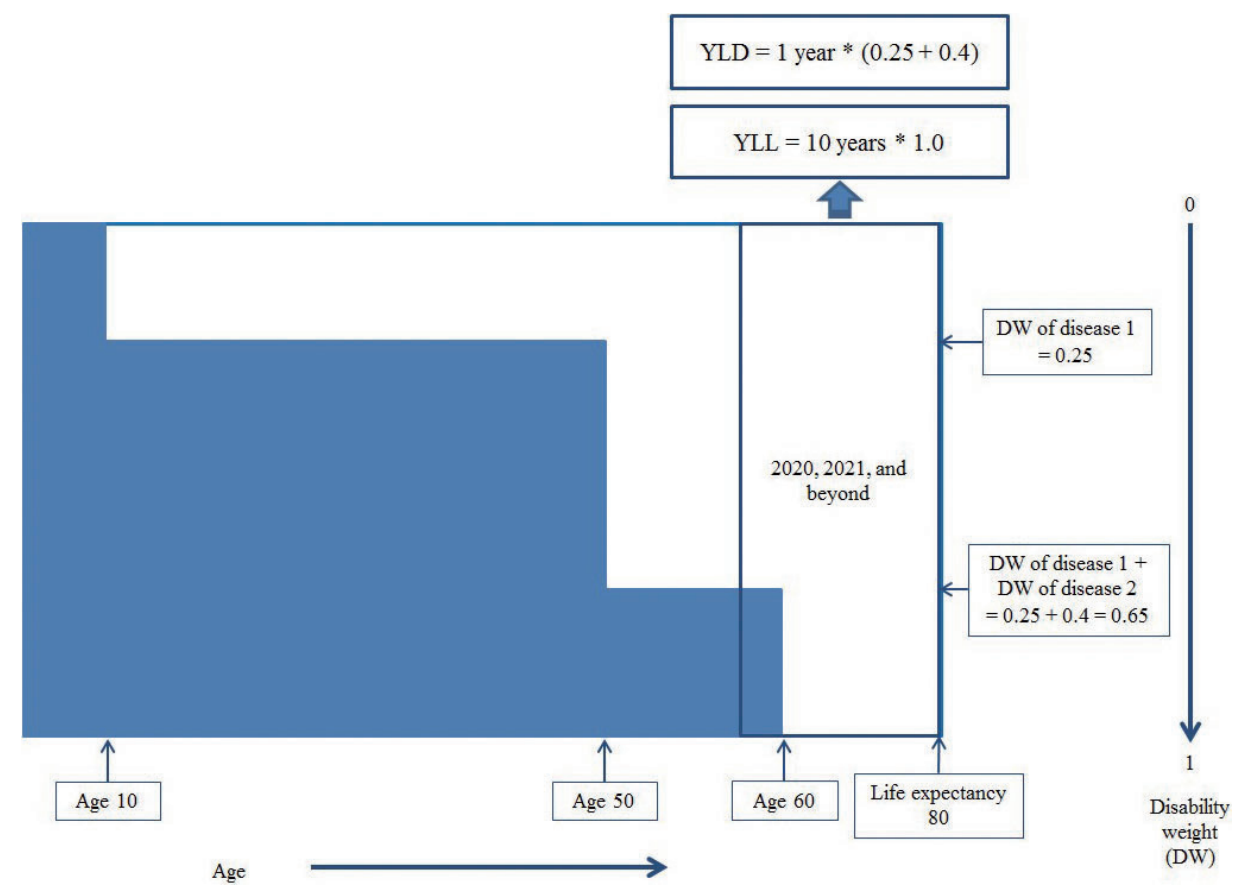

Figure 4. An example of disability-adjusted life-year estimation using the hybrid approach. YLD, years lost due to disability; YLL, years of life lost. 
Table 1. Methodological advantages and disadvantages of DALY estimation approaches

\begin{tabular}{|c|c|c|}
\hline Variables & Advantages & Disadvantages \\
\hline Incidence-based approach & $\begin{array}{l}\text { YLD and YLL are measured consistently } \\
\text { A more valid data source is provided for policies or } \\
\text { interventions that focus on disease prevention }\end{array}$ & $\begin{array}{l}\text { It is difficult to accurately measure the burden of diseases showing } \\
\text { a fall in incidence but a persistently high level of prevalence } \\
\text { YLD estimation is influenced by the method of applying the discount } \\
\text { rate } \\
\text { Data on disease duration are required } \\
\text { The incorporation of comorbidities is relatively difficult }\end{array}$ \\
\hline $\begin{array}{l}\text { Prevalence-based approach } \\
\text { (with a focus on the hybrid } \\
\text { approach) }\end{array}$ & $\begin{array}{l}\text { YLD estimation is not significantly influenced by the } \\
\text { method of applying the discount rate } \\
\text { YLD estimation does not require data on disease duration } \\
\text { YLD estimation can more easily consider comorbidities }\end{array}$ & $\begin{array}{l}\text { YLD and YLL measurements may cause confusion, as they rely on } \\
\text { different methods } \\
\text { For diseases with a short duration, such as infectious diseases, YLD } \\
\text { may be underestimated }\end{array}$ \\
\hline
\end{tabular}

DALY, disability-adjusted life-years; YLD, years lost due to disability; YLL, years of life lost.

Table 1 summarizes the methodological advantages and disadvantages of each approach. First, the incidence-based approach is advantageous in that YLD and YLL are measured consistently. For decision-making in public healthcare with a focus on disease prevention, DALY estimations made using the incidence-based approach are likely to provide a more valid data source. The drawback, however, is the quantification of diseases showing a gradual fall in incidence but a persistent high prevalence. The incidence-based approach is also vulnerable to time-related problems, as its estimated values are significantly influenced by the method used to apply the discount rate and disease duration. In addition, although there are cases where a person develops only 1 disease, since several diseases often occur simultaneously or sequentially, the incidence-based approach may also encounter difficulties in calculating the burden of disease with consideration of comorbidities.

On the contrary, the hybrid approach is relatively less influenced by the discount rate. A notable advantage of the hybrid approach is that disease duration need not be estimated. In many cases, disease duration is indirectly measured using various epidemiological data such as incidence and mortality. Indeed, in a country or region with insufficient data, it is difficult to estimate disease duration and confirm its validity [14]. YLD under the hybrid approach quantifies the level of difficulty due to the disease without considering disease duration, for which reason it is an intuitive metric. Moreover, the hybrid approach makes it easier to consider comorbidities in the estimation for YLD than the incidence-based approach.

Nevertheless, as YLD and YLL are measured using different methods in the hybrid approach, conceptual confusion may be caused for newcomers to the DALY concept. Another drawback of the hybrid approach is that the burden of diseases with a short duration may be inadequately incorporated. In other words, estimating the prevalence of infectious diseases with a short duration is difficult. For YLD estimated using the hybrid approach, care should thus be taken to avoid potential underestimation of the burden of infectious diseases.

\section{MAIN RESEARCH TRENDS IN DISABILITY-ADJUSTED LIFE-YEAR ESTIMATION APPROACHES IN KOREA AND OVERSEAS}

\section{Characteristics of Overseas Studies}

The GBD studies are the best-known studies of the burden of disease based on DALY. The 1990 GBD study led to a continuous flow of studies from the 1999 GBD study to the 2000, 2001, 2002, and 2004 studies [3,4]. In terms of the DALY estimation approach, the GBD studies have traditionally applied the incidence-based approach; however, in 2010, the methodology switched to the prevalence-based approach due to the shortcomings of incidence-based approach [10]. Specifically, the GBD studies have since applied the hybrid approach. In the 2010 GBD study, DALYs were estimated for 291 diseases across 198 countries. However, by September 2021, the DALYs of up to 369 diseases across 204 countries could be estimated [15].

Since the 2010 GBD study first applied the hybrid approach, the most notable feature of the DALY estimation approach adopted by GBD studies is the active use of the concepts of sequelae and the health state. Under the presumption that the burden of disease generally arises from its after-effects rather than the disease directly causing disabilities, GBD studies have defined the after-effects of each disease. In the 1990 GBD study, up to 483 sequelae were defined, and the scope was extended to 1160 sequelae in the 2010 GBD study [11]. 
The concept of the health state was introduced to map the sequelae and diseases, as it is difficult to set a DW for each sequela [16]. The health state incorporates data based on symptoms and the loss of certain functions, with DWs estimated and defined for each health state. In the 2010 GBD study, there were 220 health states, which steadily increased to 234 in the 2017 GBD study [17].

By introducing the concepts of sequelae and the health state into the hybrid approach, a GBD estimation system was established that could bridge the disease-sequela-health state link. This is advantageous in that a consistent GBD estimation system may be retained even if the scope of disease is extended. It is also significant that the DW estimation, which previously depended on the preferences of experts, can now reflect the preference of the general population including patients [9]. One reason for studying the burden of disease is to gather evidence for prioritizing resource allocation, and for this, it is certainly more reasonable to consider the opinions of the general public than those of a small group of experts.

However, using sequelae in the hybrid approach to estimate DALY has limitations. One may question whether the prevalence of sequelae is indeed measured based on sufficient data sources despite the steady specification of sequelae in the GBD studies. While the measurement of disease prevalence is more convenient than that of incidence, data to measure the prevalence of sequelae - if the unit of measurement is set as the sequela-are often unavailable. Thus, there is a concern about cases in which only sequelae with available data sources for measurements are included in prevalence measurements rather than all sequelae caused by the disease, which may be underestimated for rare diseases. In many cases, as the boundary between a disease and its sequelae is unclear, the ranking of the burden of disease may change according to the categorization of the cause of sequelae. In addition, since many policies in the healthcare sector are centered on disease, doubts may arise as to whether the burden of disease should be measured using the stage of sequelae.

\section{Characteristics of Studies in Korea}

Studies in Korea mainly estimated the burden of certain diseases until 2002, when the burden of disease for the Korean population was estimated based on DALYs to develop a framework for NBD studies [18]. DALY rankings for 21 diseases have been proposed in some studies, which have applied the incidence-based approach in accordance with the World Health
Organization methodology. Afterward, the 2007 NBD study in Korea focused on chronic diseases, excluding infectious diseases and injuries, using the incidence-based approach as applied in previous studies [19].

As in GBD studies, the 2012 study was the turning point in NBD studies in Korea. This study estimated DALYs for 313 diseases based on the methodology used in the 2010 GBD study $[20,21]$. The 2012 NBD study adopted the disease classification system of the 2010 GBD study but did not use the age weighting or discount rate. In addition, the 2012 NBD study applied paired comparisons as the valuation method for calculating and using DW per disease, following the 2010 GBD study [22]. However, from the perspective of DALY estimation, the 2012 NBD study in Korea retained the incidence-based approach, unlike the $2010 \mathrm{GBD}$ study, because of the insufficient data on sequelae with epidemiological analyses in the disease classification system of the 2010 GBD study. For example, in the GBD study, the sequelae of chronic renal failure were specified according to cause (e.g., diabetes, hypertension), whereas in Korea, the epidemiological data used to deduce the prevalence of the sequelae of chronic renal failure were insufficient at the time.

However, attempts have been made to estimate DALYs using the hybrid approach, as in the 2010 GBD study. Specifically, a base to access the classification system bridging the diseasesequelae -health state link was established by defining the DWs for 258 health states, as in the 2010 GBD study [23]. In addition, DALYs were estimated using the hybrid approach for cancer, a disease with relatively sufficient data sources [24]. In particular, the distribution of sequelae was estimated using the Surveillance, Epidemiology, and End Results stage data of the Korea Central Cancer Registry.

After the 2012 NBD study, DALYs were estimated for 260 diseases between 2007 and 2015 in the 2015 NBD study [25]. As in the 2012 NBD study, DALYs in the 2015 NBD study were estimated using the incidence-based approach. A study compared the DALYs estimated for injuries using the incidence-based approach with the DALYs estimated using the hybrid approach [26]. The study used the data from the Korean National Hospital Discharge Survey to estimate DALYs related to injuries in 2014, showing that all estimation approaches could calculate valid DALY results with sufficient data sources. Nevertheless, the main DALY estimation approach in Korea remains the incidence-based approach. 


\section{FUTURE PROJECTS TO PROMOTE RESEARCH ON VARIOUS DISABILITY-ADJUSTED LIFE-YEAR ESTIMATION APPROACHES}

If GBD studies adopt the hybrid approach as the main DALY estimation method, overseas NBD studies are likely to follow that trend. Moreover, if NBD studies in Korea likewise apply the prevalence-based approach, including the hybrid approach, more vigorously to estimate DALY, the potential for between-study comparisons of the burden of disease may increase. The construction of epidemiological data should precede DALY estimation studies using more varied approaches. As in GBD studies, the resulting data should contain both diseases and sequelae to allow the evaluation of the burden of disease caused by specific sequelae. On the contrary, for the sequelae determined to be critical, it is necessary to establish a nationwide system of data collection for subsequent estimations.

However, a fundamental consideration of the continuous specification of sequelae, as in GBD studies, also seems necessary. In lieu of the categorization of sequelae, the severity of a disease itself may be categorized to estimate YLD as the product of a disease with a specific severity and its respective DW. In this case, a prerequisite is collecting distribution data for the disease severity and DW of each disease with a categorized severity $[27,28]$; the probability of data construction seems higher than collecting data on categorized sequelae. In addition, a study has applied the quality-adjusted life-year as a summary measure of population health to measure the burden of disease as the product of health-related quality of life and disease prevalence [29]. Nonetheless, such studies should also be preceded by the construction of valid epidemiological data. Specifically, more epidemiological studies on severity distribution using health-related quality of life measures or clinical indicators need to be conducted.

Which DALY estimation approach is superior is difficult to clarify because each approach has advantages and disadvantages [13]. Thus, a suitable approach should be selected in accordance with the purpose of estimating the burden of disease. It is particularly important to clearly state the selected approach for the DALY estimation in each study. It is thus necessary to develop a reporting guideline for such studies to improve communication among researchers as well as to facilitate the quality evaluation of studies of the burden of disease.

\section{CONCLUSION}

This study analyzed 3 DALY estimation approaches-namely, the incidence-based approach, the pure prevalence-based approach, and the hybrid approach. Each approach has advantages and disadvantages, and the choice of a method depends on the purpose of the study of the burden of disease and the planned use of the study's outcomes. The GBD studies, which previously estimated DALYs using the incidencebased approach, switched to using the hybrid approach in 2010, while NBD studies in Korea still mainly apply the incidence-based approach. More detailed data sources should be established before conducting studies using more varied DALY estimation approaches, and a practical measure would be to develop a methodology for estimating DALYs based on disease severity rather than the sub-categorization of sequelae. The findings of this study should help researchers decide which estimation approach to use in studies of the burden of disease to enhance the validity of DALY estimation methods.

\section{Ethics Statement}

Ethical review and approval were waived for this study, because this study was not not involving humans or animals.

\section{CONFLICT OF INTEREST}

The authors have no conflicts of interest associated with the material presented in this paper.

\section{FUNDING}

This research was supported by a grant of the Korea Health Technology R\&D Project through the Korea Health Industry Development Institute (KHIDI), funded by the Ministry of Health \& Welfare, Republic of Korea (grant No. HI18C0446).

\section{ACKNOWLEDGEMENTS}

The funding agency had no role in the study design, analysis and interpretation of the data, or the preparation of the manu script. 


\section{AUTHOR CONTRIBUTIONS}

Conceptualization: Kim YE, Jung YS, Ock M, Yoon SJ. Data curation: Kim YE, Ock M. Funding acquisition: Yoon SJ. Methodology: Kim YE, Jung YS, Ock M, Yoon SJ. Project administration: Jung YS. Visualization: Ock M. Writing - original draft: Kim YE, Ock M. Writing - review \& editing: Kim YE, Jung YS, Ock M, Yoon SJ.

\section{ORCID}

$\begin{array}{ll}\text { Young-Eun Kim } & \text { https://orcid.org/0000-0003-0694-6844 } \\ \text { Yoon-Sun Jung } & \text { https://orcid.org/0000-0002-9379-4908 } \\ \text { Minsu Ock } & \text { https://orcid.org/0000-0001-9949-9224 } \\ \text { Seok-Jun Yoon } & \text { https://orcid.org/0000-0003-3297-0071 }\end{array}$

\section{REFERENCES}

1. Jung YS, Kim YE, Park H, Oh IH, Jo MW, Ock M, et al. Measuring the burden of disease in Korea, 2008-2018. J Prev Med Public Health 2021;54(5):293-300.

2. World Bank. World development report 1993: investing in health. 16th ed. New York: Oxford University Press, 1993, p. 1-16.

3. Murray CJ, Lopez AD. Regional patterns of disability-free life expectancy and disability-adjusted life expectancy: global burden of disease study. Lancet 1997;349(9062):1347-1352.

4. Murray CJ, Lopez AD. Global mortality, disability, and the contribution of risk factors: Global Burden of Disease Study. Lancet 1997;349(9063):1436-1442.

5. Murray CJ, Lopez AD. Alternative projections of mortality and disability by cause 1990-2020: Global Burden of Disease Study. Lancet 1997;349(9064):1498-1504.

6. Hyder AA, Puvanachandra P, Morrow RH. Measuring the health of populations: explaining composite indicators. J Public Health Res 2012;1(3):222-228.

7. Lopez AD, Mathers CD, Ezzati M, Jamison DT, Murray CJ. Global burden of disease and risk factors; 2006 [cited 2021 Oct 1]. Available from: https://documents1.worldbank.org/curated/ en/251181468140658232/pdf/364010PAPEROGI101OFFICIAL OUSE0ONLY1.pdf.

8. Devleesschauwer B, Havelaar AH, Maertens de Noordhout C, Haagsma JA, Praet N, Dorny P, et al. Calculating disability-adjusted life years to quantify burden of disease. Int J Public Health 2014;59(3):565-569.

9. Ock M, Ko S, Lee HJ, Jo MW. Review of issues for disability weight studies. Health Policy Manag 2016;26(4):352-358 (Korean).

10. Murray CJ, Ezzati M, Flaxman AD, Lim S, Lozano R, Michaud C, et al. GBD 2010: design, definitions, and metrics. Lancet 2012; 380(9859):2063-2066.

11. Vos T, Flaxman AD, Naghavi M, Lozano R, Michaud C, Ezzati M, et al. Years lived with disability (YLDs) for 1160 sequelae of 289 diseases and injuries 1990-2010: a systematic analysis for the Global Burden of Disease Study 2010. Lancet 2012;380 (9859):2163-2196.

12. Lim SS, Vos T, Flaxman AD, Danaei G, Shibuya K, Adair-Rohani $\mathrm{H}$, et al. A comparative risk assessment of burden of disease and injury attributable to 67 risk factors and risk factor clusters in 21 regions, 1990-2010: a systematic analysis for the Global Burden of Disease Study 2010. Lancet 2012;380(9859):22242260.

13. Schroeder SA. Incidence, prevalence, and hybrid approaches to calculating disability-adjusted life years. Popul Health Metr 2012;10(1):19.

14. Cho SW, Kim SH, Kim YE, Yoon SJ, Jo MW. Estimating lifetime duration of diabetes by age and gender in the Korean population using a markov model. J Korean Med Sci 2019;34(Suppl 1):e74.

15. GBD 2019 Diseases and Injuries Collaborators. Global burden of 369 diseases and injuries in 204 countries and territories, 1990-2019: a systematic analysis for the Global Burden of Disease Study 2019. Lancet 2020;396(10258):1204-1222.

16. Salomon JA, Vos T, Hogan DR, Gagnon M, Naghavi M, Mokdad $A$, et al. Common values in assessing health outcomes from disease and injury: disability weights measurement study for the Global Burden of Disease Study 2010. Lancet 2012;380 (9859):2129-2143.

17. GBD 2017 Disease and Injury Incidence and Prevalence Collaborators. Global, regional, and national incidence, prevalence, and years lived with disability for 354 diseases and injuries for 195 countries and territories, 1990-2017: a systematic analysis for the Global Burden of Disease Study 2017. Lancet 2018; 392(10159):1789-1858.

18. Yoon SJ, Bae SC, Lee SI, Chang H, Jo HS, Sung JH, et al. Measuring the burden of disease in Korea. J Korean Med Sci 2007; 22(3):518-523.

19. Oh IH, Yoon SJ, Kim EJ. The burden of disease in Korea. J Korean Med Assoc 2011;54(6):646-652 (Korean).

20. Yoon J, Seo H, Oh IH, Yoon SJ. The non-communicable disease burden in Korea: findings from the 2012 Korean Burden of Disease Study. J Korean Med Sci 2016;31(Suppl 2):S158-S167. 
21. Lee YR, Moon K, Kim YA, Park SY, Oh CM, Lee KS, et al. Disability-adjusted life years for communicable disease in the Korean Burden of Disease Study 2012. J Korean Med Sci 2016;31(Suppl 2):S178-S183.

22. Ock M, Lee JY, Oh IH, Park H, Yoon SJ, Jo MW. Disability weights measurement for 228 causes of disease in the Korean Burden of Disease Study 2012. J Korean Med Sci 2016;31(Suppl 2): S129-S138.

23. Ock M, Ahn J, Yoon SJ, Jo MW. Estimation of disability weights in the general population of South Korea using a paired comparison. PLoS One 2016;11(9):e0162478.

24. Gong YH, Yoon SJ, Jo MW, Kim A, Kim YA, Yoon J, et al. The burden of cancer in Korea during 2012: findings from a prevalencebased approach. J Korean Med Sci 2016;31(Suppl 2):S168-S177.

25. Kim YE, Park H, Jo MW, Oh IH, Go DS, Jung J, et al. Trends and patterns of burden of disease and injuries in Korea using disability-adjusted life years. J Korean Med Sci 2019;34(Suppl 1): e75.

26. Park B, Park B, Lee WK, Kim YE, Yoon SJ, Park H. Incidence-based versus prevalence-based approaches on measuring disabilityadjusted life years for injury. J Korean Med Sci 2019;34(Suppl 1):e69.

27. Ock M, Jo MW, Gong YH, Lee HJ, Lee J, Sim CS. Estimating the severity distribution of disease in South Korea using EQ-5D3L: a cross-sectional study. BMC Public Health 2016;16:234.

28. Ock M, Park B, Park H, Oh IH, Yoon SJ, Cho B, et al. Disability weights measurement for 289 causes of disease considering disease severity in Korea. J Korean Med Sci 2019;34(Suppl 1): e60.

29. Ock M, Han JW, Lee JY, Kim SH, Jo MW. Estimating quality-adjusted life-year loss due to noncommunicable diseases in Korean adults through to the year 2040. Value Health 2015;18(1): 61-66. 